\title{
Cardiovascular impairment in young adults horizontally infected with HIV-1 in childhood
}

\author{
Florentina Dumitrescu ${ }^{1,2^{*}}$, Dina Maria Cupşa ${ }^{2}$, Andreea Cristina Stoian ${ }^{1,2}$, Augustin Cupşa $a^{1,2}$, Irina Niculescu ${ }^{1,2}$, \\ Lucian Giubelan ${ }^{1,2}$, Daniela Culman² \\ From The 9th Edition of the Scientific Days of the National Institute for Infectious Diseases Prof Dr Matei Bals \\ Bucharest, Romania. 23-25 October 2013
}

\section{Background}

We aimed to evaluate the prevalence and spectrum of cardiovascular impairment (CVI) in young HIV/AIDS patients in evidence at the HIV/AIDS Craiova Regional Center.

\section{Methods}

We performed a retrospective study between 01 January 1996 - 31 December 2005 and a prospective study between 01 June 2005 - 30 June 2010; it targeted epidemiological issues, the clinical spectrum of CVI in patient with horizontal HIV infection, acquired during 1987-1990 (lot P) compared to patients with other transmission routes of infection (lot $S$ ).

\section{Results}

CVI was encountered in $61(10.23 \%)$ of 596 patients parenterally-infected with HIV-1 in childhood and $32(16.16 \%)$ of 198 patients infected through sexual route, with statistically significant differences in the prevalence of CVI depending on the transmission route of HIV-1 $\left(\mathrm{pChi}^{2}=0.02\right)$, probably being underestimated in pre-HAART era. The average age at the moment of CVI was $9.02 \pm 4.76$ years in lot $P$ vs. $41.47 \pm 12.41$ years in lot $\mathrm{S}$ ( $\mathrm{p}<0.0001)$. The spectrum of CVI was dominated by arrhythmias and conduction blocks 42 patients (45.16\%), dilated cardiomyopathy (DCM) 18 patients (19.35\%), pulmonary arterial hypertension 17 patients (18.27\%). The most common entity identified in parenterallyinfected patients in early childhood was DCM 16 patients lot $\mathrm{P}$ vs. 2 patients lot $\mathrm{S}$ ( $\mathrm{p}$ Fischer test $=0.026$ ), found in moderate and severe immunosuppressed patients, with virological failure, often associated with respiratory disease. In sexually-infected patients diseases due to atherosclerosis prevailed 17 patients ( $p$ Fisher test $<0.0001$ ). The presence of high blood pressure in 2 young people with HIV/AIDS raises the issue of cardiovascular risk and occurrence of coronary heart disease in young patients. Although the survival rate of patients with CVI (15.21 \pm 0.62 years) was similar to that of the patients without CVI (13.78 \pm 0.25 years), for patients with CVI, death due to direct cardiac damage was significant: 9 patients (52.94\%).

\section{Conclusion}

The prevalence of CVI was low, probably overlooked and underdiagnosed, at least in early ages and young adults, dominated by arrhythmias and DCM; monitoring and management in individual care programs constitutes a way for improving the quality of life and prognosis for people with HIV/AIDS.

\section{Authors' details}

'University of Medicine and Pharmacy Craiova, Romania. "2"Victor Babeş" Clinical Hospital of Infectious Diseases and Pneumology, Craiova, Romania.

Published: 16 December 2013

doi:10.1186/1471-2334-13-S1-028

Cite this article as: Dumitrescu et al:: Cardiovascular impairment in young adults horizontally infected with HIV-1 in childhood. BMC Infectious Diseases 2013 13(Suppl 1):O28. 